\title{
Machine Learning Based Diagnostics of Developmental Coordination Disorder using Electroencephalographic Data
}

\author{
Ricardo Buettner \\ Aalen University \\ Germany \\ ricardo.buettner@hs- \\ aalen.de
}

\author{
Michael Buechele \\ Aalen University \\ Germany \\ michael.buechele@hs- \\ aalen.de
}

\author{
Benedikt Grimmeisen \\ Aalen University \\ Germany \\ benedikt.grimmeisen@hs- \\ aalen.de
}

\author{
Patrick Ulrich \\ Aalen University \\ Germany \\ patrick.ulrich@hs- \\ aalen.de
}

\begin{abstract}
We report on promising results concerning the fast and accurate diagnosis of developmental coordination disorder (DCD) which heavily impacts the life of affected children with emotional and behavioral issues. Using a machine learning classifier on spectral data of electroencephalography (EEG) recordings and unfolding the traditional frequency bandwidth in a fine-graded equidistant 99-point spectrum we were able to reach an accuracy of over 99.35 percent having only one misclassification. Our machine learning work contributes to healthcare and information systems research. While current diagnostic methods in use are either complicated, time-consuming, or inaccurate, our automated machine-based approach is accurate and reliable. Our results also provide more insights into the relationship between DCD and brain activity which could stimulate future work in medicine.
\end{abstract}

\section{Introduction}

Developmental coordination disorders (DCD) are a subfield of motor disorders affecting children all around the world with a prevalence of 1.8 percent [1]. Since DCD is treatable and the negative impact on motoric abilities can be reduced by an early-stage diagnosis, a fast and reliable diagnosis system for the citizens would have a strong impact $[2,3]$.

According to the Diagnostic and Statistical Manual of Mental Disorders DSM-5, DCD is defined as a motor disorder resulting in clumsiness, slowness, and inaccuracy in a dimension "significantly and persistently interfering with activities of daily living appropriate to chronological age and impacts academic/school productivity, prevocational and vocational activities, leisure, and play," while not being attributable to intellectual, visual or neurological conditions [4].

DCD has a major impact on children in terms of limiting them in their educational and social life and having negative effects on their health $[5,6]$. They are observed as having over five times more emotional and behavioral problems and with a significantly lower health-related quality of life compared to their peers $[5,6]$. Children with DCD are at higher risk of developing symptoms of anxiety and depression than their typically developing peers [7]. There is a significant impact on educational achievement and therefore life chances for adolescents with DCD due to problems with reading skills, social communication difficulties, and hyperactivity, and inattention [8].

While DCD is affecting children all around the world with a prevalence of about 1.8 , regionally prevalence is up to 17.9 percent $[1,9-13]$.

Questionnaires for mass screenings failed very often in terms of accuracy and reliability [14-16].

However, driven by increases in computational power and the availability of huge new datasets, ITbased healthcare has undergone a dramatic upswing in the past years [17]. The field has witnessed spectacular advances in the ability of machines to understand data and this can be accompanied by extraordinary successes in medicine, in particular for diagnosing diseases $[18,19]$ or detecting higher orders of medical substance interactions [20]. The application of most modern machine learning using big data within the healthcare domain fosters this success [18-25].

To help detect DCD reliably and early, we propose a machine learning based algorithm for diagnosing DCD, which surpasses current methods in terms of accuracy and reliability.

The most important contributions are:

1) We built a highly effective classification model diagnosing DCD with a balanced accuracy of over 99.35 percent. 
2) Our model substantially outperforms all existing approaches, including questionnairebased instruments in medical use.

3) The approach is fast, robust, reliable, and independent from human influences.

4) Our results on higher brain activation in Upper-Theta and Lower-Alpha EEG subbands for DCD patients deepen understanding of the theory that people with DCD use different brain regions than control peers to support their motor performance and which is reflected in these specific EEG subspectra (novel contribution to brain activation theory).

The paper is organized as follows: next, we present an overview of currently used methods for DCD diagnostics, the progress of automatic detection of DCD, and the exemplary progress of automatic detection of other disorders, diseases, and addictions. After that the research methodology is divided a) to give information about the dataset used, and b) to describe our algorithm. Then we show the machine learning results regarding the performance evaluation and analysis of the EEG frequency bands. After discussing the results, we conclude our research while reflecting on the limitations of our work and providing suggestions for future research.

\section{Theoretical background}

There are five different approaches to motor assessment and treatment for children with DCD. These are to use different explanatory frameworks (Normative Functional Skill Approach, General Abilities Approach, Neurodevelopmental Theory, Dynamical Systems Theory, and the Cognitive Neuroscientific Approach) [2]. Based on these, several different diagnostic methods were derived. The current gold standard in diagnostics of DCD are standardized tests which allow manual assessments of motor skills. The Bruininks-Oseretsky Test of Motor Proficiency, Second Edition (BOT-2), and the Movement Assessment Battery for Children, Second Edition (MABC-2) represent the two most commonly used examples [26, 27]. There the children participate in a set of age-specific exercises and their motorcoordinative abilities are assessed within the three motoric development dimensions of manual dexterity, ball skills, and balance. Although both tests have a high reliability between 78 and 97 percent as well as a consistency above 90 percent [15, 28, 29], these kinds of test require highly qualified practitioners, like occupational or physical therapists who also have to be familiar with statistical concepts to perform and evaluate the test correctly and achieve representative results. Even for experienced operators, an entire assessment including preparation, administration, processing, and evaluation takes at least 90 minutes [15].

These motoric tests also tend to be very pricy with basic sets around $\$ 1,100$ each. When using the BOT2 , the following limitation must also be taken into account, that the norms used for the evaluation of the results are not generally valid but are reflective of the current demographics in the United States [15, 28, 29]. Due to the time consuming and expensive characteristics of these tests, some experts tend to use motoric questionnaires like the DCD-Q [30], CSAPPA [31], or M-ABC-Checklist which must be answered either by the child, its parents, or its teachers. As questionnaires filled in by parents or teachers (e.g. DCD-Q, M-ABC-Checklist) focus on rating motorcoordinative abilities with the three motoric development dimensions and the daily activities [2], the questionnaires filled in by children (e.g. CSAPPA, All about Me Scale [32]) also focus on the selfperceptions of the children, like confidence in walking or self-esteem. While these tests offer a good idea of how the child perceives the disorder itself [33], this self-assessment has proven to be neither specific nor sensitive enough [2]. Therefore, these questionnaires are just used as an initial step in the diagnostics of DCD.

All these more traditional approaches tend to have a solid accuracy as well as consistency, and they also require a fair amount of time, money, and qualifications in order to be utilized. Thus, an automated method overcoming these limitations would be beneficial to diagnostics in DCD. Since the availability of electronic health data is increasing, this trend yields an opportunity for models and algorithms backed by Machine Learning to use their strength in detecting patterns in data to improve traditional healthcare $[17,18]$. Previous studies on risk prediction of infections, outcomes of diseases, or diagnosis of symptoms have proven the capabilities of Machine Learning in Healthcare with great accuracy and levels of performance [17]. In the more specific domain of DCD, Martinez-Manzanera et. al. [34] used the Random Forest method in differential diagnostics to automatically detect ataxia and DCD. Sensor units provided motoric movement data on children with ataxia, DCD, and a control group executing an upper limb coordination task. While ataxia (74.4 percent) and the controls (87.4 percent) could be classified well, DCD could only be detected with an accuracy of 24.8 percent on average [34].

However, Machine Leaning was also used in the domain of EEG analysis: Different researchers 
developed algorithms based on brain activity using EEG recordings that were able to predict disorders like alcoholism [22] or schizophrenia [35], detect epileptic seizures [36, 37], and classify sleep stages [38] faster and more accurately than human doctors. Driven by these interesting results, further Machine Learning related research on theories explaining the relationships between DCD and brain activity can benefit. For example, Zwicker et al. [39, 40] found indications for such correlations such as differing patterns in brain activities of healthy and developmentally challenged children during finemotor tasks or learning new skills. A deep understanding of the neurobiology of DCD, as well as strong evidence for a correlation between brain activity and DCD, are still absent.

\section{Method}

This research follows the Design Science methodology [41]. Our contribution is an IT-artifact to classify DCD on EEG data. The IT-artifact is an algorithm, rigorously evaluated by ten-fold crossvalidation.

\subsection{Dataset}

The dataset was provided by Vařeka et al. [42] and is publicly available without restrictions. It was created in 2013 at the University of West Bohemia of Pilsen (Czech Republic). It contains the EEG-data of 32 school-age participants (age: 7-10 years; 11 females and 21 male) each 6-7 minutes long. The EEG data was acquired using 19 electrodes arranged according to the standard 10-20 system [43], with a sampling frequency set to $1 \mathrm{kHz}$ and cut-off frequencies of 0.1 and $250 \mathrm{~Hz}$. Each time series includes 1,000 samples per second with a resolution of $0.1 \mu \mathrm{V}$.

All participants suffered from impaired hearing and were divided into three groups. The MABC-2 motor test provided an overall score ranging from 0 to 100. Based on the individual results, the subjects were separated into three groups according to the traffic light system of Henderson et al. [27]: Subjects with a score above 67 were labeled as having no movement difficulties (green light). Subjects with an overall score between 67 and 57 were rated as having a risk of a movement difficulty (yellow light) and subjects with scores below 57 were labeled as having movement difficulties (red light). Sixteen of the subjects were in the green area and served as the control group, 4 were in the yellow area and 12 subjects scored within the red zone.

Each subject completed 600 tests in total, separated in two equal runs of 300 stimulations. This stimulation was multimodal, meaning both auditory and visual, in which the sound of one of three animals (Goat, Dog, Cat) was synchronized with the picture of the animal. Using one of the animals (goat), always occurring with the matching sound and picture as reference or standard stimulus, additional rare stimuli were utilized to trigger a specific task. The task of each subject was to reply to the target stimulus by pressing one of two buttons attached to the armrests of the chair. One button was dedicated to a matching stimulus, where the sound of the animal matched the picture shown. The second button indicated a stimulus where the picture did not match the sound heard.

The dataset also provides additional metadata including used hardware, used stimulation protocol, and detailed information about each participant of the study. The research group not only recorded the data but also validated and evaluated the quality of the recordings of each participant. In total the elicitation as well as the data itself are of impeccable quality.

\subsection{Machine Learning Method}

This part of the paper covers the pre-processing of the given data, the feature extraction in the form of the spectral analysis, and the classification as well as the validation of the created model (see Figure 1).

Pre-processing: In a first step, the four subjects with a MABC-2 motorically score inside the yellow zone according to the traffic light system were removed from the dataset since they could not clearly be assigned to either the control group or the group suffering from a developmental disorder.

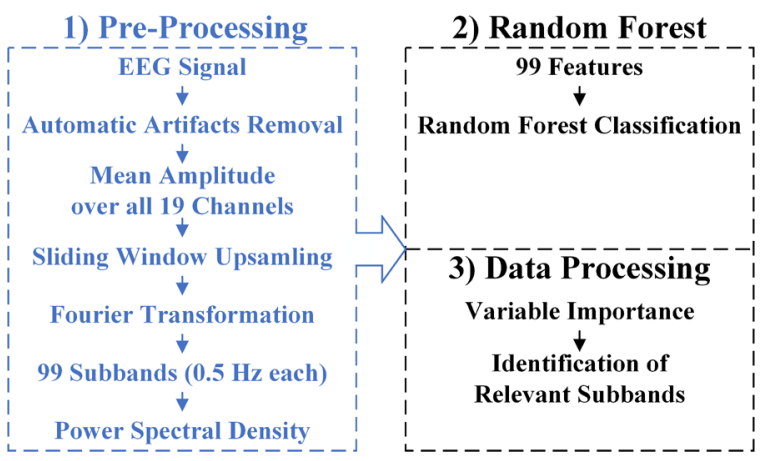

Figure 1: Processing steps

Based on the 28 available seven-minute recordings, we applied a sliding window approach, partitioning the data into one-minute windows with a 30 -second overlap. As suggested by Bell et al. [44], the linear decomposition approach was used to partially correct the EEG recordings since all three requirements described by Bell et al. [44] are fulfilled for EEG data 
in general. Since the electrodes attached to the scalp have to record the finest signals from the brain, mixed signals as well as disturbances like eye movement, muscle activities, and blinking are found in the data [45]. By utilizing the independent component analysis (ICA) any noise was detected and afterward removed.

Spectral analysis and feature extraction: After preparing and cleaning the records of any noise, possible features could be extracted. Initially, Feature Engineering was applied to each sample in the form of dimensional reduction by calculating the mean over all 19 channels of the original signal. To determine the activity of the brain represented by the EEG data [45, 46], the responsible frequencies leading to the time series data had to be extracted. Therefore, the time domain of the samples was transformed into the frequency domain by utilizing the Fast-Fourier Transformation as a method for estimating the distribution of the frequency contained in the EEG recordings [47, 48]. Instead of using the classic division of the frequency bands into alpha, beta, theta, delta, and gamma $[45,46]$ we unfolded the band reaching from 0 up to $50 \mathrm{~Hz}$ in steps of $0.5 \mathrm{~Hz}$ [49]. According to this approach, the information content of finer frequency bands was proven to be higher than the broad bands used in the classic division. Therefore, the resulting frequencies and corresponding amplitudes of the spectral analysis were separated in 99 bins, each covering a range of $0.5 \mathrm{~Hz}$ with the cumulated amplitude for this frequency area.

Classification: To answer the question of whether it is possible to separate the healthy control group from the patients suffering from DCD, and if yes to determine which frequency bands are the most important. We used a Random Forest to generate multiple distinct decision trees whose terminal leaves represent the two classes DCD and Non-DCD. During training, spectral power thresholds of randomly selected sub-bands are calculated according to the class of an individual sample which are used to add decision nodes to the tree. When testing, a majority vote of the individual decision trees in the random forest is used to determine the final class of every sample. Originally proposed by Breiman [50] in 2001, Random Forest still stands out today due to its highly effective processing of large amounts of data and its excellent accuracy $[22-25,36]$ and performs very well with EEG data [51], especially in combination with the fine-grained EEG spectrum [22-25, 36]. It is also possible to draw direct conclusions about the most important variables for classification. We used the caret package in $\mathrm{R}$ to implement the classification using a common Training/Test Split of $75 \%$ and $25 \%$ of the recordings with 10 repetitions each using $\mathrm{n}=$ 100 trees. After training and testing our specific Random Forest the variable importance used internally by the classifier could be extracted and observed. Each of the variables in the resulting data represents the statistical significance of a specific frequency band related to the influence on the model.

Validation: We validated the classifier with a tenfold cross-validation. The accuracy of the model and further metrics were calculated and presented (see Table 1), as well as a confusion matrix (see Table 2).

\section{Results}

For the training of Random Forest, we applied the caret v6.0-82 package within an $\mathrm{R}$ x64 3.5.1 environment running on a 16 GB RAM custom workstation. The training ran in a k-fold cross validation style on the training data with 10 iterations. During training, the test data was not shown to the model. The Random Forest classifier was built using 500 voting trees.

\subsection{Performance evaluation}

We evaluated the classifier in terms of accuracy, sensitivity (true positive rate), specificity (true negative rate), precision (positive predictive value), negative predictive value), Cohen's Kappa score, and balanced accuracy.

Table 1 shows the excellent performance of our classifier with an accuracy of 99.47 percent in detecting DCD. Both true negative rate and positive predictive values are 100 percent. The true positive rate is 98.7 percent, and the negative predictive value is 99.12 percent. Prevalence is 40.74 percent. Cohen'sKappa score is 98.9 percent.

\begin{tabular}{lr}
\hline Performance indicator & Value \\
\hline Accuracy & $99.47 \%$ \\
True positive rate & $98.70 \%$ \\
True negative rate & $100 \%$ \\
Positive predictive value & $100 \%$ \\
Negative predictive value & $99.12 \%$ \\
Prevalence & $40.74 \%$ \\
Balanced accuracy & $99.35 \%$ \\
Kappa & $98.90 \%$ \\
\hline
\end{tabular}

Table 1: Evaluation indicators 
The confusion matrix (see Table 2) points out that there is only one misclassification, which is in the form of a false negative.

\begin{tabular}{|c|c|c|c|}
\hline & \multicolumn{2}{|c|}{ Predicted } \\
\hline & & DCD & Non-DCD \\
\hline \multirow{2}{*}{ 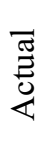 } & DCD & 76 & 1 \\
\hline & $\begin{array}{l}\text { Non- } \\
\text { DCD }\end{array}$ & 0 & 112 \\
\hline
\end{tabular}

Table 2: Confusion matrix of our classifier

\subsection{Importance of specific frequency bands}

The trained Random Forest classifier can be analyzed to gain information about the impact of specific frequency bands (variable importance) on the prediction. Figure 2 shows the variable importance of the $0.5 \mathrm{~Hz}$ frequency bands with the band between 6.5 and $7 \mathrm{~Hz}$ having the highest impact representing 100 percent. The importance of the remaining frequency bands was subtracted relative to this baseline. Having importance of 90.96 percent, the second runner-up frequency band was between 5.5 and $6 \mathrm{~Hz}$ followed by the band between 7 and $7.5 \mathrm{~Hz}$ having an importance of 82.70 percent. The remaining frequency bands in the top ten do have an importance as follows: $9-9.5$
$\mathrm{Hz} 81.37$ percent, $8.5-9 \mathrm{~Hz} 77.48$ percent, $7.5-8 \mathrm{~Hz}$ 70.55 percent, $4.5-5 \mathrm{~Hz} 69.58$ percent, $6-6.5 \mathrm{~Hz}$ 69.23 percent, $9.5-10 \mathrm{~Hz} 68.33 \mathrm{~Hz}$ and the band between 5 and $5.5 \mathrm{~Hz}$ having an importance of 64.60 percent. In contrast, the band between 40 and $40.5 \mathrm{~Hz}$ has no impact on the decision tree with an importance of 0 percent. Overall, 82 of 99 frequency bands were below the level of importance of $50 \%$.

In a subsequent procedure, the mean power value for the ten most important frequency bands was calculated for both classes, DCD, and control, using the results of the spectral analysis (see Table 3). Utilizing students' two-sample t-tests with spectral data of the ten most important frequency bands as samples, we were able to revise if the real difference in the population's power mean value exists and if yes if it is significant. We defined the null hypothesis $H_{0}$ : $\mu_{\text {Healthy }}-\mu_{D C D}=0$ where $\mu_{\text {Healthy }}$ is the populations mean in the power of all healthy children and $\mu_{D C D}$ is the populations mean in the power of all children suffering from DCD, saying that there is no difference in the mean of both populations. Further, we defined the alternative hypothesis as $H_{0}$ : $\mu_{\text {Healthy }}-\mu_{D C D} \neq 0$ for a two-sided test saying there is a difference in the mean value of the two populations either being positive or negative. Test statistics are calculated by the t-Test. The resulting p-values for each of the ten tested frequency bands are shown in Table 3 . The null hypothesis $H_{0}$ could be rejected for

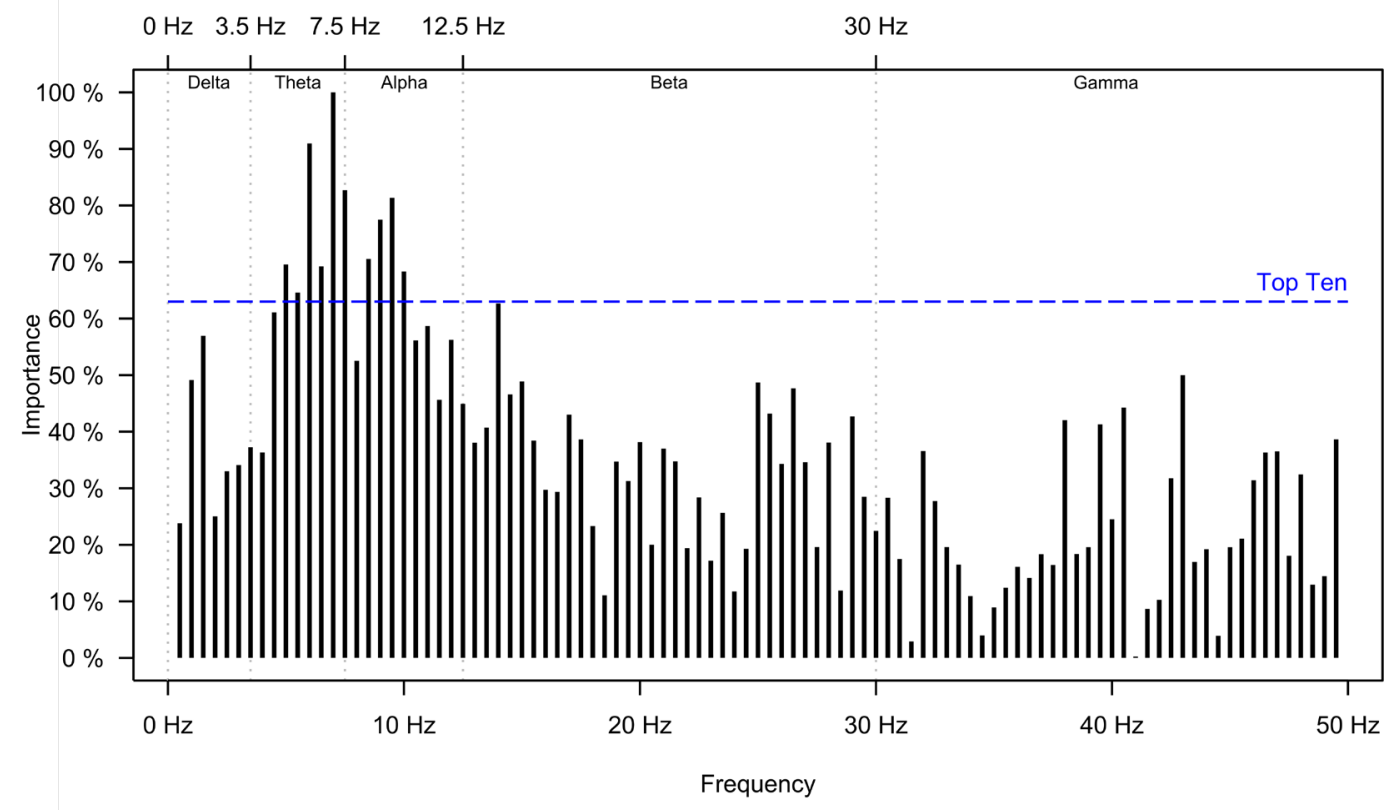

Figure 2: Variable importance of the $0.5 \mathrm{~Hz}$ frequency bins in relation to the standard EEG bandwidths 
all ten frequency bands with a confidence over 95 percent since all p-values for $H_{0}$ were below 0.05 . Except for the lowest band between 4.5 - and $5 \mathrm{~Hz}$ the confidence in rejecting $H_{0}$ and accepting the alternative hypothesis $H_{0}$ is well above 99 percent $\left(H_{0}\right.$ p-value $<0.01)$.

\begin{tabular}{|c|c|c|c|c|}
\hline Frequency & $\begin{array}{c}\text { Power } \\
\text { Control }\end{array}$ & $\begin{array}{c}\text { Power } \\
\text { DCD }\end{array}$ & $\begin{array}{c}\text { p- } \\
\text { value }\end{array}$ & $\begin{array}{c}\text { Cohen's } \\
\text { d }\end{array}$ \\
\hline $\mathbf{9 . 5 - 1 0 ~ H z}$ & 0.067368 & 0.080711 & $<0.001$ & 0.30 \\
\hline $\mathbf{9 - 9 . 5 ~ H z}$ & 0.066915 & 0.077106 & $<0.01$ & 0.24 \\
\hline $\mathbf{8 . 5 - 9} \mathbf{~ H z}$ & 0.064018 & 0.075975 & $<0.001$ & 0.30 \\
\hline $\mathbf{7 . 5 - 8} \mathbf{~ H z}$ & 0.064493 & 0.076674 & $<0.001$ & 0.32 \\
\hline $\mathbf{7 - 7 . 5 ~ H z}$ & 0.070555 & 0.084613 & $<0.001$ & 0.33 \\
\hline $\mathbf{6 . 5 - 7} \mathbf{~ H z}$ & 0.073230 & 0.089196 & $<0.001$ & 0.33 \\
\hline $\mathbf{6 - 6 . 5 ~ H z}$ & 0.072716 & 0.086109 & $<0.001$ & 0.30 \\
\hline $\mathbf{5 . 5 - 6 ~ H z}$ & 0.077876 & 0.087262 & $<0.01$ & 0.22 \\
\hline $\mathbf{5 - 5 . 5 ~ H z}$ & 0.078451 & 0.088366 & $<0.01$ & 0.24 \\
\hline $\mathbf{4 . 5}-\mathbf{5 H z}$ & 0.089544 & 0.096962 & $<0.05$ & 0.15 \\
\hline
\end{tabular}

Table 3: Analysis of the ten most important bands

To validate the outcome of the two-sample t-test as well as measure the selectivity and effect size of the statistical method we calculated Cohen's d for each of the ten most important frequency bands.

As shown in Table 3 the frequency bands between 6.5 and $8 \mathrm{~Hz}$ have the highest effect size. The mean in effect sizes over all ten most important frequency bands for DCD classification is 0.27 .

\section{Discussion}

This paper hypothesized that machine learning technologies yield an opportunity to provide diagnostics in the domain of DCD. Several scientific reviews criticized traditional methods like manual tests for being time-consuming with a minimum duration of 90 minutes even for experienced practitioners, being expensive at a base-set price of over $\$ 1,100$, and being subjective as a result of their dependence on the children and the practitioners. Cheaper and faster alternatives like questionnaires are not accurate enough to deliver reliable diagnoses. Manzanera et al. [34] suggested machine learningbased approaches, which didn't even perform as well as the already inaccurate questionnaires. In contrast to his approach utilizing motoric movement data like the traditional approaches, our approach was in focusing on the speculated difference in brain activity of healthy and DCD affected children. Underpinned by the theoretical work of Zwicker et al. [39] which suspected that brain activities between these two groups might be different, our model was indeed able to classify subjects suffering from DCD with a balanced accuracy of 99.35 percent using EEG recordings of the brain activity. Looking at this outstanding performance with only one single misclassification has proven us to be correct: a machine learning based classification of DCD is possible and even outperforms traditional approaches.

As a result of the successful model, we were able to determine the ten most important frequency bands that were able to distinguish between DCD affected and healthy children. While using the traditional frequency bands might only have led us to a range of important bands between theta and alpha spanning a range of $9 \mathrm{~Hz}$, the 99-band approach enabled us to narrow this range down to a range of $5 \mathrm{~Hz}$, half the size between upper theta and lower alpha. It was also possible to distinguish the fine-grade bands inside each traditional band as shown in Fig. 1 to examine their influence in detail.

Using the statistical methods of two-sample t-test as well as Cohen's d we were able to confirm the theory by Zwicker et al [39] about the difference in brain activity: The average power of the two groups in the ten most important frequency bands shown in table 3 were significantly different, proving that there is indeed a difference in brain activity. As the results show, the children suffering from DCD displayed greater activation in all these ten bands. This higher load in activation eventually leads to the described "cognitive fatigue" [39] of children suffering from DCD in executing motor movements like the tasks in the experiment of the dataset used. The results of Cohen's d with a mean in effect size of 0.27 also show differing brain activity based on the power means.

The greater activity in the traditional theta and alpha band might give some explanation of the slowness of DCD affected children described in the literature. While these two bands are considered characteristic of sleep phases or a relaxed state of mind in healthy children [52], DCD affected children had higher activations of these bands during mental workload through the stimuli and related motoric tasks of the experiment. All frequency bands inside the Beta and Gamma band, which are typically activated during stress, inner restlessness, and high concentration [52], have an importance below 50 percent or even below 10 percent. Since children suffering from DCD are motorically impaired it might imply that they must concentrate more to complete a motoric task like pushing the correct button in the case of the experiment, which is not the case, based on the data shown in Figure 2. 


\section{Conclusion}

Though a correlation between DCD and brain activity has already been indicated [39, 40], machinedriven research on this topic was absent. Multiclassification of motor disorders performed weakly on DCD [34] driven by movement data, only reaching 24.8 percent accuracy. With our algorithm, we were able to reliably detect DCD in children with only one misclassification.

This research pursued the question of whether machine learning technologies in DCD detection can provide diagnostics. The results of our classifier demonstrate this question has to be answered as true.

Our classifier could detect DCD in children in milliseconds, using seven minutes [46] of EEG data per participant, compared to manual testing methods with a duration of 90 minutes, if done quickly [15].

As a result, DCD diagnostics may break with the expensiveness, the lengthiness, the high expertise requirements, and the subjectivity of current, manual state-of-the-art testing methods.

Additionally, being able to reliably detect DCD on EEG data, this research can confirm a correlation between DCD in children and brain activity.

Furthermore, our findings of the relevance of each frequency band can enable more focused and specific research on DCD and thereby better understand this disorder.

\subsection{Limitations}

The ten-fold cross-validation provides high internal validity. However, the external validity has yet to be evaluated. Replication studies using other datasets with different participants are needed to add an external layer of validation.

The IT artifact has been executed detached from the clinical application field. To validate the algorithm against specific and possible unknown influences and circumstances, an implementation in a clinical environment must be done.

\subsection{Future Work}

In future work, we will re-evaluate our results on different data sets to increase the external validity of our DCD detection algorithm. Therefore, we will use a data set with different participants.

Besides, in future work, we will triangulate EEG sensor data with other physiological sensor data $[25$, 53-59]. While we successfully evaluated our novel ML approach on several diseases [23, 24, 49, 53, 6063], addictions [64], stress [65] and personality traits
[66], we will experimentally evaluate whether our approach is also robust under various conditions of a user's cognitive workload [67-69], concentration [70] and mindfulness $[71,72]$ by physicians and patients in multi-agent settings [73-76].

To further evaluate the model, we will benchmark our Random Forest algorithm to other ML approaches, including convolutional neural networks [21, 77-79] and other ML methods like XGBoost [80] or support vector machines [81] on brain activities in the identified areas for detecting EDS [62] and further research on EEG data

Furthermore, we will implement the IT artifact in a clinical environment. Figure 3 shows the related framework used for detection, treatment, and evaluation. By using this model, clinicians could use existing EEG data of a patient to provide an initial indicator for a possible diagnosis instead of performing time-consuming manual tests and so reduce the workload of the medical staff.

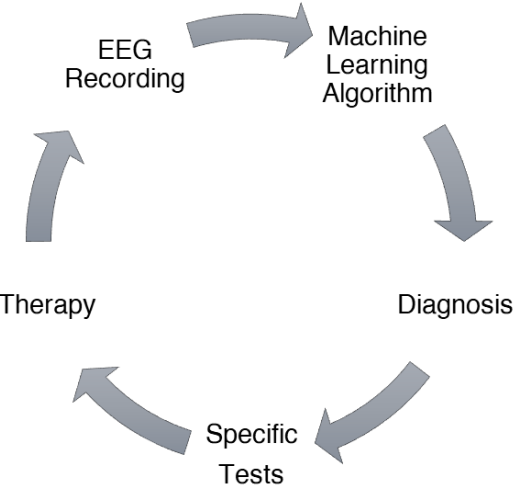

Figure 3: DCD treatment-diagnose circle

We will evaluate the technology acceptance $[56,82-$ $85]$ and trust [86, 87], as it is crucial for real-world scenarios.

\section{Acknowledgements}

This research is funded by the Carl Zeiss Foundation and the German Federal Ministry of Education and Research (13FH4E03IA, 13FH4E07IA, 13FH176PX8, 13FH4E05IA).

\section{References}

[1] M.-H. Tseng, C.-P. Fu, B. N. Wilson, and F.-C. Hu, "Psychometric properties of a Chinese version of the Developmental Coordination Disorder Questionnaire in community-based children," Res Dev Disabil, vol. 31, no. 1, pp. 33-45, 2010.

[2] M. M. Schoemaker, B. Flapper, N. P. Verheij, B. N. Wilson, H. A. Reinders-Messelink, and A. de Kloet, "Evaluation of the Developmental Coordination Disorder Questionnaire as a screening instrument," 
Dev Med Child Neurol, vol. 48, no. 8, pp. 668-673, 2006.

[3] P. H. Wilson, "Practitioner review: approaches to assessment and treatment of children with DCD: an evaluative review," J Child Psychol Psychiatry, vol. 46, no. 8, pp. 806-823, 2005.

[4] American Psychiatric Association, Diagnostic and Statistical Manual of Mental Disorders: DSM-5, 5th ed.: American Psychiatric Publishing, 2013.

[5] L. Crane, E. Sumner, and E. L. Hill, "Emotional and behavioural problems in children with Developmental Coordination Disorder: Exploring parent and teacher reports," Res Dev Disabil, vol. 70, pp. 67-74, 2017.

[6] H. C. Karras, D. N. Morin, K. Gill, S. IzadiNajafabadi, and J. G. Zwicker, "Health-related quality of life of children with Developmental Coordination Disorder," Res Dev Disabil, vol. 84, pp. 85-95, 2019.

[7] T. T. G. Draghi, J. L. Cavalcante Neto, L. A. Rohr, L. D. Jelsma, and E. Tudella, "Symptoms of anxiety and depression in children with developmental coordination disorder: a systematic review," J Pediatr (Rio J), vol. 96, no. 1, pp. 8-19, 2020.

[8] I. Harrowell, L. Hollén, R. Lingam, and A. Emond, "The impact of developmental coordination disorder on educational achievement in secondary school," Res Dev Disabil, vol. 72, pp. 13-22, 2018.

[9] R. Lingam, L. Hunt, J. Golding, M. Jongmans, and A. Emond, "Prevalence of developmental coordination disorder using the DSM-IV at 7 years of age: a UK population-based study," Pediatrics, vol. 123, no. 4, e693-700, 2009.

[10] G. D. Tsiotra et al., "A comparison of developmental coordination disorder prevalence rates in Canadian and Greek children," J Adolesc Health, vol. 39, no. 1, pp. 125-127, 2006.

[11] L. Delgado-Lobete, S. Santos-Del-Riego, S. PértegaDíaz, and R. Montes-Montes, "Prevalence of suspected developmental coordination disorder and associated factors in Spanish classrooms," Res Dev Disabil, vol. 86, pp. 31-40, 2019.

[12] B. Kadesjö and C. Gillberg, "Developmental coordination disorder in Swedish 7-year-old children," J Am Acad Child Adolesc Psychiatry, vol. 38, no. 7, pp. 820-828, 1999.

[13] K. K. H. Chung, "Understanding developmental dyslexia in Chinese: linking research to practice," APJDD, vol. 4, no. 1, pp. 3-15, 2016.

[14] J. Hua et al., "The reliability and validity of the Developmental Coordination Disorder Questionnaire'07 for children aged 4-6 years in mainland China," Res Dev Disabil, vol. 47, pp. 405415, 2015.

[15] J. C. Deitz, D. Kartin, and K. Kopp, "Review of the Bruininks-Oseretsky Test of Motor Proficiency, Second Edition (BOT-2)," Phys Occup Ther Pediatr, vol. 27, no. 4, pp. 87-102, 2007.

[16] Y.-P. Wuang et al., "Game-Based Auxiliary Training System for improving visual perceptual dysfunction in children with developmental disabilities: A proposed design and evaluation," Computers \& Education, vol. 124, pp. 27-36, 2018.
[17] K. Tsoi, S. Poon, and P. Hung, "Introduction to the Minitrack on Big Data on Healthcare Application," in HICSS-51 Proc., 2018.

[18] A. Esteva et al., "A guide to deep learning in healthcare," Nat Med, vol. 25, no. 1, pp. 24-29, 2019.

[19] C. Poon et al., "Derivation and Analysis of Dynamic Handwriting Features as Clinical Markers of Parkinson's Disease," in HICSS-52 Proc., 2019.

[20] S. K. Poon et al., "A novel approach in discovering significant interactions from TCM patient prescription data," Int J Data Min Bioinform, vol. 5, no. 4, pp. 353368, 2011.

[21] J. Gross, J. Breitenbach, H. Baumgartl, and R. Buettner, "High-Performance Detection of Corneal Ulceration Using Image Classification with Convolutional Neural Networks," in HICSS-54 Proc., 2021, in Press.

[22] T. Rieg, J. Frick, M. Hitzler, and R. Buettner, "Highperformance detection of alcoholism by unfolding the amalgamated EEG spectra using the Random Forests method," in HICSS-52 Proc., 2019, pp. 3769-3777.

[23] R. Buettner, A. Grimmeisen, and A. Gotschlich, "High-performance Diagnosis of Sleep Disorders: A Novel, Accurate and Fast Machine Learning Approach Using Electroencephalographic Data," in HICSS-53 Proc., 2020, pp. 3246-3255.

[24] R. Buettner, D. Beil, S. Scholtz, and A. Djemai, "Development of a machine learning based algorithm to accurately detect schizophrenia based on oneminute EEG recordings," in HICSS-53 Proc., 2020, pp. 3216-3225.

[25] R. Buettner, M. Hirschmiller, K. Schlosser, M. Roessle, M. Fernandes, and I. J. Timm, "Highperformance exclusion of schizophrenia using a novel machine learning method on EEG data," in IEEE Healthcom 2019 Proc., 2019, pp. 1-6.

[26] R. H. Bruininks, K. Steffens, A. Spiegel, and J. K. Werder, "The Bruininks-Oseretsky test of motor proficiency: development, research, and intervention strategies," in 2nd International Symposium Psychomotor Therapy and Adapted Physical Activity, 1989.

[27] S. E. Henderson, A. David, and A. B. Sugden, Movement Assessment Battery for Children-2 second edition [Movement ABC-2]: The Physical Corporation, 2007.

[28] Y.-P. Wuang and C.-Y. Su, "Reliability and responsiveness of the Bruininks-Oseretsky Test of Motor Proficiency-Second Edition in children with intellectual disability," Res Dev Disabil, vol. 30, no. 5, pp. 847-855, 2009.

[29] A. Griffiths, R. Toovey, P. E. Morgan, and A. J. Spittle, "Psychometric properties of gross motor assessment tools for children: a systematic review," BMJ Open, vol. 8, no. 10, e021734, 2018.

[30] B. N. Wilson, S. G. Crawford, D. Green, G. Roberts, A. Aylott, and B. J. Kaplan, "Psychometric properties of the revised Developmental Coordination Disorder Questionnaire," Phys Occup Ther Pediatr, vol. 29, no. 2, pp. 182-202, 2009. 
[31] S. Rosenblum, "The development and standardization of the Children Activity Scales (ChAS-P/T) for the early identification of children with Developmental Coordination Disorders," Child Care Health Dev, vol. 32, no. 6, pp. 619-632, 2006.

[32] C. Missiuna, "Development of "All about Me," a Scale That Measures Children's Perceived Motor Competence," The Occupational Therapy Journal of Research, vol. 18, no. 2, pp. 85-108, 1998.

[33] J. A. Hay, R. Hawes, and B. E. Faught, "Evaluation of a screening instrument for developmental coordination disorder," J Adolesc Health, vol. 34, no. 4, pp. 308-313, 2004.

[34] O. Martinez-Manzanera et al., "Instrumented fingerto-nose test classification in children with ataxia or developmental coordination disorder and controls," Clin Biomech, vol. 60, pp. 51-59, 2018.

[35] J. Frick, T. Rieg, and R. Buettner, "Detection of schizophrenia: a machine learning algorithm for potential early detection and prevention based on event-related potentials," in HICSS-54 Proc., 2021, in Press.

[36] R. Buettner, J. Frick, and T. Rieg, "High-performance detection of epilepsy in seizure-free EEG recordings: A novel machine learning approach using very specific epileptic EEG sub-bands," in ICIS 2019 Proc., 2019, pp. 1-16.

[37] T. Rieg, J. Frick, and R. Buettner, "Machine learningbased diagnosis of epilepsy in clinical routine: Lessons learned from a retrospective pilot study," in Information Systems and Neuroscience: NeuroIS Retreat 2020, 2020, pp. 276-283.

[38] N. Michielli, U. R. Acharya, and F. Molinari, "Cascaded LSTM recurrent neural network for automated sleep stage classification using singlechannel EEG signals," Comput Biol Med, vol. 106, pp. 71-81, 2019.

[39] J. G. Zwicker, C. Missiuna, S. R. Harris, and L. A. Boyd, "Brain activation of children with developmental coordination disorder is different than peers," Pediatrics, vol. 126, no. 3, e678-86, 2010.

[40] J. G. Zwicker, C. Missiuna, S. R. Harris, and L. A. Boyd, "Developmental coordination disorder: A review and update," Eur J Paediatr Neurol, vol. 16, no. 6, pp. 573-581, 2012.

[41] A. R. Hevner, S. T. March, J. Park, and S. Ram, "Design Science in Information Systems Research," MIS Quarterly, vol. 28, no. 1, pp. 75-105, 2004.

[42] L. Vařeka, P. Bruha, R. Moucek, P. Mautner, L. Cepicka, and I. Holecková, "Developmental coordination disorder in children - experimental work and data annotation," GigaScience, vol. 6, no. 4, pp. 1-6, 2017.

[43] H. H. Jasper, "The Ten-Twenty Electrode System of the International Federation," Electroencephalogr Clin Neurophysiol, vol. 10, pp. 371-375, 1958.

[44] A. J. Bell and T. J. Sejnowski, "An InformationMaximization Approach to Blind Separation and Blind Deconvolution," Neural Comput, vol. 7, no. 6, pp. 1129-1159, 1995.
[45] M. Teplan, "Fundamentals of EEG Measurement," Meas. Sci. Rev., vol. 2, no. 2, pp. 1-11, 2002.

[46] P. Olejniczak, "Neurophysiologic Basis of EEG," J Clin Neurophysiol, vol. 23, no. 3, pp. 186-189, 2006.

[47] M. K. van Vugt, P. B. Sederberg, and M. J. Kahana, "Comparison of spectral analysis methods for characterizing brain oscillations," $J$ Neurosci Methods, vol. 162, 1-2, pp. 49-63, 2007.

[48] M. Ahirwal and N. Londhe, "Power Spectrum Analysis of EEG Signals for Estimating Visual Attention," Int. J. Comput. Appl, vol. 42, no. 15, pp. 34-40, 2012.

[49] R. Buettner, T. Rieg, and J. Frick, "Machine Learning Based Diagnosis of Diseases Using the Unfolded EEG Spectra: Towards an Intelligent Software Sensor," in Information Systems and Neuroscience: NeuroIS Retreat 2019, 2019, pp. 165-172.

[50] L. Breiman, "Random Forests," Machine Learning, vol. 45, no. 1, pp. 5-32, 2001.

[51] L. Fraiwan, K. Lweesy, N. Khasawneh, H. Wenz, and H. Dickhaus, "Automated sleep stage identification system based on time-frequency analysis of a single EEG channel and random forest classifier," Comput Methods Programs Biomed, vol. 108, no. 1, pp. 1019, 2012.

[52] W. J. Freeman and R. Q. Quiroga, Imaging Brain Function With EEG: Advanced Temporal and Spatial Analysis of Electroencephalographic Signals: Springer, 2013.

[53] R. Buettner, J. Fuhrmann, and L. Kolb, "Towards high-performance differentiation between Narcolepsy and Idiopathic Hypersomnia in 10 minute EEG recordings using a Novel Machine Learning Approach," in IEEE Healthcom 2019 Proc., 2019, pp. $1-7$.

[54] R. Buettner, L. Bachus, L. Konzmann, and S. Prohaska, "Asking Both the User's Heart and Its Owner: Empirical Evidence for Substance Dualism," in Information Systems and Neuroscience: NeuroIS Retreat '18, 2018, pp. 251-257.

[55] A. Eckhardt et al., "Objective measures of IS usage behavior under conditions of experience and pressure using eye fixation data," in ICIS 2013 Proc., 2013.

[56] R. Buettner, B. Daxenberger, A. Eckhardt, and C. Maier, "Cognitive Workload Induced by Information Systems: Introducing an Objective Way of Measuring based on Pupillary Diameter Responses," in Pre-ICIS HCI/MIS 2013 Proceeding, 2013, Paper 20.

[57] R. Buettner, "Social Inclusion in E-Participation and E-Government Solutions: A Systematic Laboratoryexperimental Approach Using Objective Psychophysiological Measures," in EGOV/ePart 2013 Proc., 2013, pp. 260-261.

[58] R. Buettner, S. Sauer, C. Maier, and A. Eckhardt, "Real-time Prediction of User Performance based on Pupillary Assessment via Eye-Tracking," AIS Trans Hum-Comput Interact, vol. 10, no. 1, pp. 26-60, 2018.

[59] R. Buettner, "Robust user identification based on facial action units unaffected by users' emotions," in HICSS-51 Proc., 2018, pp. 265-273. 
[60] D. Raab, H. Baumgartl, and R. Buettner, "Machine Learning Based Diagnosis of Binge Eating Disorder Using EEG Recordings," in PACIS '20 Proc., 2020.

[61] H. Baumgartl, F. Dikici, D. Sauter, and R. Buettner, "Detecting Antisocial Personality Disorder Using a Novel Machine Learning Algorithm Based on Electroencephalographic Data," in PACIS '20 Proc., 2020.

[62] J. Breitenbach, H. Baumgartl, and R. Buettner, "Detection of Excessive Daytime Sleepiness in Resting-State EEG Recordings: A Novel Machine Learning Approach Using Specific EEG Sub-Bands and Channels," in AMCIS 2020 Proc., 2020.

[63] H. Baumgartl, P. Roessler, D. Sauter, and R. Buettner, "Measuring Social Desirability Using a Novel Machine Learning Approach Based on EEG Data," in PACIS '20 Proc., 2020.

[64] J. Gross, H. Baumgartl, and R. Buettner, "A Novel Machine Learning Approach for High-Performance Diagnosis of Premature Internet Addiction Using the Unfolded EEG Spectra," in AMCIS 2020 Proc., 2020.

[65] H. Baumgartl, E. Fezer, and R. Buettner, "Two-level classification of chronic stress using machine learning on resting-state EEG recordings," in AMCIS 2020 Proc., 2020.

[66] H. Baumgartl, S. Bayerlein, and R. Buettner, "Measuring extraversion using EEG data," in Information Systems and Neuroscience: NeuroIS Retreat 2020, 2020, pp. 259-265.

[67] R. Buettner, I. F. Scheuermann, C. Koot, M. Rössle, and I. J. Timm, "Stationarity of a User's Pupil Size Signal as a Precondition of Pupillary-Based Mental Workload Evaluation," in Information Systems and Neuroscience: NeuroIS Retreat, 2018, pp. 195-200.

[68] R. Buettner, "The Relationship Between Visual Website Complexity and a User's Mental Workload: A NeuroIS Perspective," in Information Systems and Neuroscience: Gmunden Retreat on NeuroIS 2016, 2016, pp. 107-113.

[69] R. Buettner, "A user's cognitive workload perspective in negotiation support systems: An eye-tracking experiment," in PACIS 2016 Proc., 2016, Paper 115.

[70] R. Buettner, H. Baumgartl, and D. Sauter, "Microsaccades as a Predictor of a User's Level of Concentration," in Information Systems and Neuroscience: NeuroIS Retreat, 2018, pp. 173-177.

[71] S. Sauer, J. Lemke, W. Zinn, R. Buettner, and N. Kohls, "Mindful in a random forest: Assessing the validity of mindfulness items using random forests methods," Pers. Individ. Differ., vol. 81, pp. 117-123, 2015.

[72] S. Sauer, R. Buettner, T. Heidenreich, J. Lemke, C. Berg, and C. Kurz, "Mindful Machine Learning," Eur J Psychol Assess, vol. 34, no. 1, pp. 6-13, 2018.

[73] R. Buettner, "A Systematic Literature Review of Crowdsourcing Research from a Human Resource Management Perspective," in HICSS-48 Proc., 2015, pp. 4609-4618.

[74] J. Landes and R. Buettner, "Job Allocation in a Temporary Employment Agency via Multi- dimensional Price VCG Auctions Using a Multi-agent System," in MICAI 2011 Proc., 2011, pp. 182-187.

[75] R. Buettner and J. Landes, "Web Service-based Applications for Electronic Labor Markets: A Multidimensional Price VCG Auction with Individual Utilities," in ICIW 2012 Proc., 2012, pp. 168-177.

[76] S. C. Rodermund, I. J. Timm, and R. Buettner, "Towards Simulation-Based Preplanning for Experimental Analysis of Nudging," in WI-2020 Proc., 2020, pp. 1219-1233.

[77] H. Baumgartl and R. Buettner, "Development of a Highly Precise Place Recognition Module for Effective Human-robot Interactions in Changing Lighting and Viewpoint Conditions," in HICSS-53 Proc., 2020, pp. 563-572.

[78] H. Baumgartl, J. Tomas, R. Buettner, and M. Merkel, "A deep learning-based model for defect detection in laser-powder bed fusion using in-situ thermographic monitoring," Prog Addit Manuf, vol. 5, no. 3, pp. 277285, 2020.

[79] R. Buettner and H. Baumgartl, "A Highly Effective Deep Learning Based Escape Route Recognition Module for Autonomous Robots in Crisis and Emergency Situations," in HICSS-52 Proc., 2019, pp. 659-666.

[80] T. Chen and C. Guestrin, "XGBoost," in KDD 2016 Proc., 2016, pp. 785-794.

[81] K. N. T. Månsson et al., "Predicting long-term outcome of Internet-delivered cognitive behavior therapy for social anxiety disorder using fMRI and support vector machine learning," Transl Psychiatry, vol. 5, no. 3, e530, 2015.

[82] R. Buettner, "Analyzing the Problem of Employee Internal Social Network Site Avoidance: Are Users Resistant due to their Privacy Concerns?," in HICSS48 Proc., 2015, pp. 1819-1828.

[83] R. Buettner, "Towards a New Personal Information Technology Acceptance Model: Conceptualization and Empirical Evidence from a Bring Your Own Device Dataset," in AMCIS 2015 Proc., 2015.

[84] R. Buettner, "Getting a Job via Career-Oriented Social Networking Sites: The Weakness of Ties," in HICSS49 Proc., 2016, pp. 2156-2165.

[85] R. Buettner, "Getting a job via career-oriented social networking markets," Electron Mark, vol. 27, no. 4, pp. 371-385, 2017.

[86] F. Meixner and R. Buettner, "Trust as an Integral Part for Success of Cloud Computing," in ICIW 2012 Proc., 2012.

[87] R. Buettner, "The impact of trust in consumer protection on internet shopping behavior: An empirical study using a large official dataset from the European Union," in BDS-2020 Proc., 2020, pp. 6669. 